http://jmscr.igmpublication.org/home/

ISSN (e)-2347-176x ISSN (p) 2455-0450

crossref DOI: https://dx.doi.org/10.18535/jmscr/v7i7.146

Journal Of Medical Science And Clinical Research

IGM Publication

An Official Publication of IGM Publication

\title{
Hepatic artery pseudoaneurysm presented with haemobilia: Rare complication of plastic biliary stent insertion
}

\author{
Authors \\ Dr Imran Sayyed*, Dr Amol Samarth \\ ${ }^{1}$ Senior Resident, ${ }^{2}$ Assistant Professor \\ Department of Gastroenterology, Government Medical College and Super Specialty Hospital, Nagpur, \\ Maharashtra-440003, India \\ *Corresponding Author \\ Dr Imran Sayyed
}

\begin{abstract}
A 45-year-old man was admitted with Obstructive jaundice. A plastic stent was placed in CBD to relieve obstructive cholangitis. Two weeks after the procedure, the patient was readmitted with cholangitis caused by hemobilia. However, USG abdomen, computed tomography (CT), endoscopic retrograde cholangiopancreatography (ERCP) failed to establish the bleeding source. Later Abdominal angiography revealed a hepatic artery pseudoaneurysm for which embolization done with Inj. Glue therapy and bleeding was stopped. Hemobilia after plastic stent placement is extremely rare. We urge Gastroenterologists to consider the possibility of a pseudoaneurysm near the stent when trying to identify the bleeding source.

Keywords: hemobilia, hepatic artery pseudoaneurysm, plastic stent.
\end{abstract}

\section{Introduction}

Hemobilia causes symptoms such as upper abdominal pain, upper gastrointestinal bleeding and jaundice, the combination of which is known as Quincke's triad due to bleeding in the bile duct. The main causes of hemobilia are iatrogenic and include liver biopsy or percutaneous biliary drainage.

Hemobilia as a complication of plastic stent placement is exceedingly rare. To the best of our knowledge, there have only been few previously reported cases. In the present case, 2 weeks after the first plastic stent placement, the patient needed to be admitted for recurrent intermittent biliary bleeding.
Several factors are associated with hemobilia after plastic stent placement. It was recently reported that endoscopic retrograde cholangiopancreatography (ERCP)-related bile duct injury, malignant tumor invasion, cholangitis, and liver abscess may contribute to hemobilia.

In the present case, ERCP induced Bile duct injury may be the cause of hemobilia.

\section{Case Report}

45 yrs old male patient from Gondhia was referred to our hospital with jaundice, patient also had itching all over body, fever and chills. later Diagnosed as case of obstructive jaundice with cholangitis and was admitted to our hospital. 
Patient is also known case of Sickle cell disease (SS Pattern).

Blood chemistry test showed that ALT was 123 IU/L, AST 145 IU/L, ALP was $651 \mathrm{U} / \mathrm{L}$, Total bilirubin was $13.3 \mathrm{mg} \%$, D. bilirubin was 10.3 gm\% Total protein was $4.8 \mathrm{gm} \%$, serum albumin was $2.5 \mathrm{gm} \%$, PT-INR 1.34, HB was $8.3 \mathrm{gm} \%$, TC-15990 with Neutrophil-80\%, platelet count134000/ul, MCV-93.1fl, HBsAg-neg, anti-HCV: neg, anti HIV-neg, Sr.Na+143, Sr.K+ 2.8, BUN30, Sr.creatinine $0.9 \mathrm{mg} \%$, RBS $-87, \mathrm{Hb}$ electrophoresis-SS pattern.

Abdomianl USG showed cholelithiasis and choledocholithiasis with CBD/IHBR dilatation, CT scan abdomen also revealed cholelithiasis and choledocholithiasis with $\mathrm{CBD} / \mathrm{IHBR}$ dilatation, we performed ERCP and CBD stones were found in common bile duct, stones were extracted from CBD (Figure 1) and plastic straight stent was inserted into CBD and later Post ERCP USG abdomen showed decompression of IHBR, pneumobilia, no stone in CBD, CBD stent in situ, sr. bilirubin also decreases and fever subsided and later patient discharged from ward.

After 15 days patient had haematemesis and malena, Jaundice and fever with chills, Later admitted to our ward, BP-was $80 / 60 \mathrm{mmHg}$, and plus rate was $110 / \mathrm{min}$, pallor was present, patient also had psoas abscess for which pitail catheter was inserted, Blood transfusions, iv fluid and iv antibiotics was given to patient, after that We carried out forward view endoscopy which shows blood in D2, Later side view endoscopy revealed bleeding from ampulla and CBD stent in ampulla (Figure 2), we carried out repeat ERCP and stent was exchanged, we placed double pigtail stent in place of straight stent and performed CT abdomen that revealed Hepatomegaly, CBD stent in situ, IHBR decompression, pneumobilia with psoas abscess and pigtail catheter in situ. But even after repeat ERCP, bleeding not controlled.

Later We performed abdominal angiography at intervention radiology department that revealed hepatic artery pseudo aneurysm (Figure 3), bleeding through CBD was noted due to fistula. fistula was formed between the hepatic artery and CBD due to straight stent that was placed into CBD earlier. That stent protruded into the CBD and was displaced with only its apex being in contact with hepatic artery and formed fistula.

After that we performed embolization therapy (with Inj. glue) on hepatic artery, to stop the bleeding, finally bleeding was stopped and patient was discharged. On follow up no further episodes of bleeding.

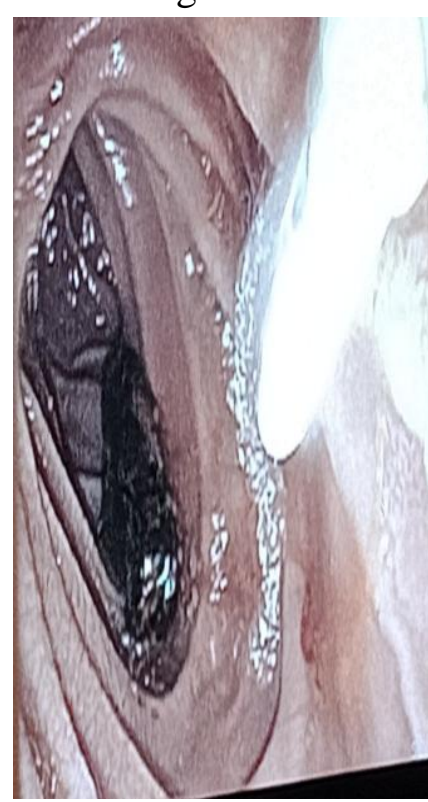

Figure 1

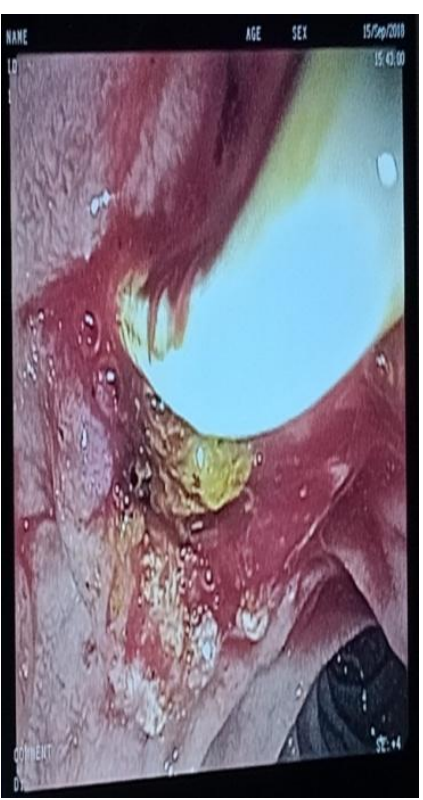

Figure 2

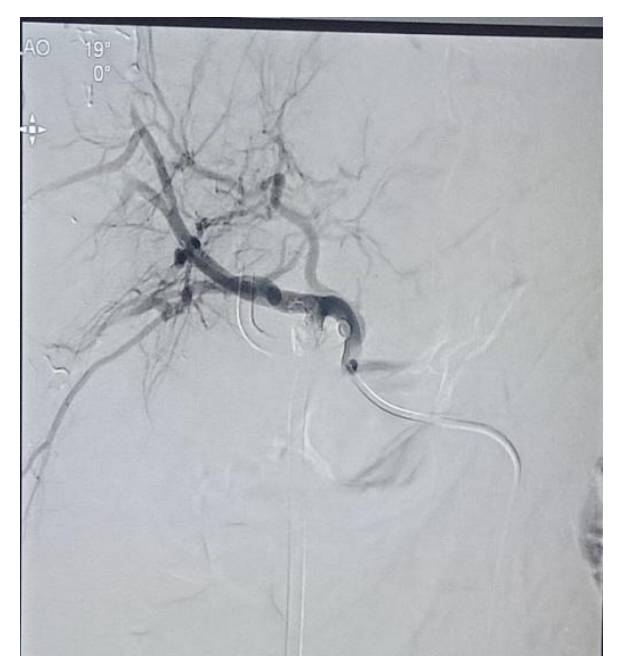

Figure 3

\section{Discussion}

Hemobilia causes symptoms, such as upper abdominal pain, upper gastrointestinal bleeding, and jaundice, the combination of which is known as Quincke's triad due to bleeding in the bile 
$\operatorname{duct}^{1}$. The main causes of hemobilia are iatrogenic and include liver biopsy or percutaneous biliary drainage $^{2}$. Apart from iatrogenic bleeding, malignant tumor is the main cause ${ }^{3}$. In this case, a hepatic artery pseudoaneurysm after plastic stent placement for Obstructive jaundice secondary to choledocholithiasis with cholangitis caused the hemobilia.

Hepatic artery pseudoaneurysm as a complication of plastic stent placement is rare. Pseudoaneurysm formation by metal stent placement for malignant biliary obstruction is reported to occur in $1.2 \%$ of cases ${ }^{4}$. In contrast to metal stents, plastic stents seem to very rarely cause pseudoaneurysm. However, the exact prevalence remains uncertain.

To the best of our knowledge, there have only been few previously reported cases of hemobilia caused by plastic stent placement ${ }^{5-9}$.

Several factors are associated with hemobilia after plastic stent placement. ERCP-related bile duct injury $^{6}$, malignant tumor invasion ${ }^{5,8}$, cholangitis ${ }^{7,8}$, and liver abscess may also ${ }^{7}$ contribute to hemobilia. Increased intra-biliary pressure and inflammation may affect the formation of aneurysms and chronic use of steroids may also contributes to formation of hepatic artery pseudoaneurysm.

In summary, we here in reported a rare case of hepatic artery pseudoaneurysm associated with a plastic stent insertion. The aneurysm was initially difficult to detect. Later detected on abdominal angiography and embolization done.

We believe that to identify the source of hemobilia, attention should be given to the possible development of a pseudoaneurysm near the plastic stent.

\section{References}

1. Chin MW, Enns R. Hemobilia. Curr Gastroenterol Rep 12: 121-129, 2010.

2. Green $\mathrm{MH}$, Duell RM, Johnson $\mathrm{CD}$, Jamieson NV. Haemobilia. Br J Surg 88: 773-786, 2001.

3. Kim KH, Kim TN. Etiology, clinical features, and endoscopic management of hemobilia: a retrospective analysis of 37 cases. Korean J Gastroenterol 59: 296-302, 2012.

4. Nezu Y, Nakaji S, Fujii H, Ishii E, Hirata N. Pseudoaneurysm caused by a selfexpandable metal stent: a report of three cases. Endoscopy 46: 248-251, 2014.

5. Conio M, Caroli-Bosc FX, Buckley M, Chiaramondia M, D’Addazio G, Munizzi F. Massive hematobilia after extraction of plastic biliary endoprosthesis. J Clin Gastroenterol 25: 706, 1997.

6. Wolters F, Ryan B, Beets-Tan R, Dejong C. Delayed massive hemobilia after biliary stenting. Endoscopy 35: 976-977, 2003.

7. Park JY, Ryu H, Bang S, Song SY, Chung JB. Hepatic artery pseudoaneurysm associated with plastic biliary stent. Yonsei Med J 48: 546-548, 2007.

8. Inoue H, Tano S, Takayama R, et al. Right hepatic artery pseudoaneurysm: rare complication of plastic biliary stent insertion. Endoscopy 43 (Suppl 2 UCTN): E396, 2011.

9. Lee SH, Hong SG, Lee KY, et al. Delayed severe hemobilia after endoscopic biliary plastic stent insertion. Clin Endosc 49: 303-307, 2016. 